\title{
A drainage catheter tangled around a closed left atrial appendage
}

\author{
Koshi Sawada $^{1} \cdot$ Hideki Kitamura $^{1}\left[\right.$ : Yutaka Koyama ${ }^{1} \cdot$ Mototsugu Tamaki $^{1} \cdot$ Yasuhiko Kawaguchi $^{1}$
}

Received: 9 March 2019 / Accepted: 7 May 2019 / Published online: 17 May 2019

(c) The Author(s) 2019

\begin{abstract}
A 70-year-old man underwent cardiac surgery including left atrial appendage closure. A pigtail catheter was inserted into the pericardial sac because of delayed tamponade. Removal of the catheter was planned for 2 days after drain insertion. However, the resistance was high and pulsatile. The patient was transferred to the catheterization laboratory and a guide wire was inserted through the catheter, revealing the catheter route around the left atrial appendage. The wire was exchanged for a stiff wire to uncurl the catheter as much as possible, then the catheter was removed. The left atrial appendage does not usually represent an obstacle to catheter removal because it is soft and shrinkable. However, once the left atrial appendage becomes closed off, it can become hard, unshrinkable and an obstacle that might be caught by the drainage catheter.
\end{abstract}

Keywords Left atrial appendage closure $\cdot$ Drainage catheter $\cdot$ Tamponade

After reports of the WATCHMAN atrial appendage closure device yielded favorable results for preventing cerebral infarction, concern about surgical left atrial appendage closure has increased [1,2]. Herein, we report a case that serves as a warning about perioperative management after surgical left atrial appendage closure.

A 70-year-old man underwent mitral valve replacement, tricuspid annuloplasty, Maze procedure and left atrial appendage closure. Intra-operative transesophageal echocardiogram demonstrated "Chicken Wing" left atrial appendage [3] with its base diameter of $25 \mathrm{~mm}$ (Fig. 1). It was closed from the inside of the atrium with a double-layered, continuous monofilament polypropylene suture. The postoperative course was uneventful, except for delayed tamponade. A pigtail catheter (Pericardiocentesis Kit; Merit Medical, USA) was inserted into the pericardial sac smoothly by echo-guided Seldinger method and pericardial effusion was drained on postoperative day 12 . The plan was to remove the catheter 2 days later. However, the resistance when removal was initiated was very high and pulsatile. As the patient complained of pain when traction was placed in the catheter, he was transferred to the catheterization laboratory.

Hideki Kitamura

kitamura@heart-center.or.jp

1 Department of Cardiovascular Surgery, Nagoya Heart Center, 1-1-14 Sunadabashi, Higashi-ku, Nagoya, Aichi 461-0045, Japan
A guide wire (Swanexcell guidewire; AuBex, Japan) was inserted through the drainage catheter, revealing that the catheter passed tortuously inside the pericardial sac and routed around the left atrial appendage (Fig. 2). The wire was exchanged for a stiff one (Amplatz Super Stiff; Boston Scientific, USA) to uncurl the catheter as much as possible, then the catheter was pulled gently and removed without any complications.

Figure 3 shows a 3-dimensional reconstruction of the plain computed tomography while the catheter was in place. We should have inserted the pigtail catheter under both echo-guided and fluoroscopic guidance to avoid too deep insertion of the catheter.

The catheter is seen going around the left atrial appendage. The normal left atrial appendage does not represent an obstacle to pulling out the catheter, because it is soft and shrinkable. However, closure can make the left atrial appendage hard and unshrinkable. Once the appendage becomes such an obstacle, the pigtail catheter can become tangled and catch on the appendage. It might happen even after the catheter-based closure. In this situation, a stiff wire is useful to uncurl the catheter and allow removal without strangling the left atrial appendage.

In cases such as the one described here, pulling forcefully on the catheter risks catastrophic consequences. 


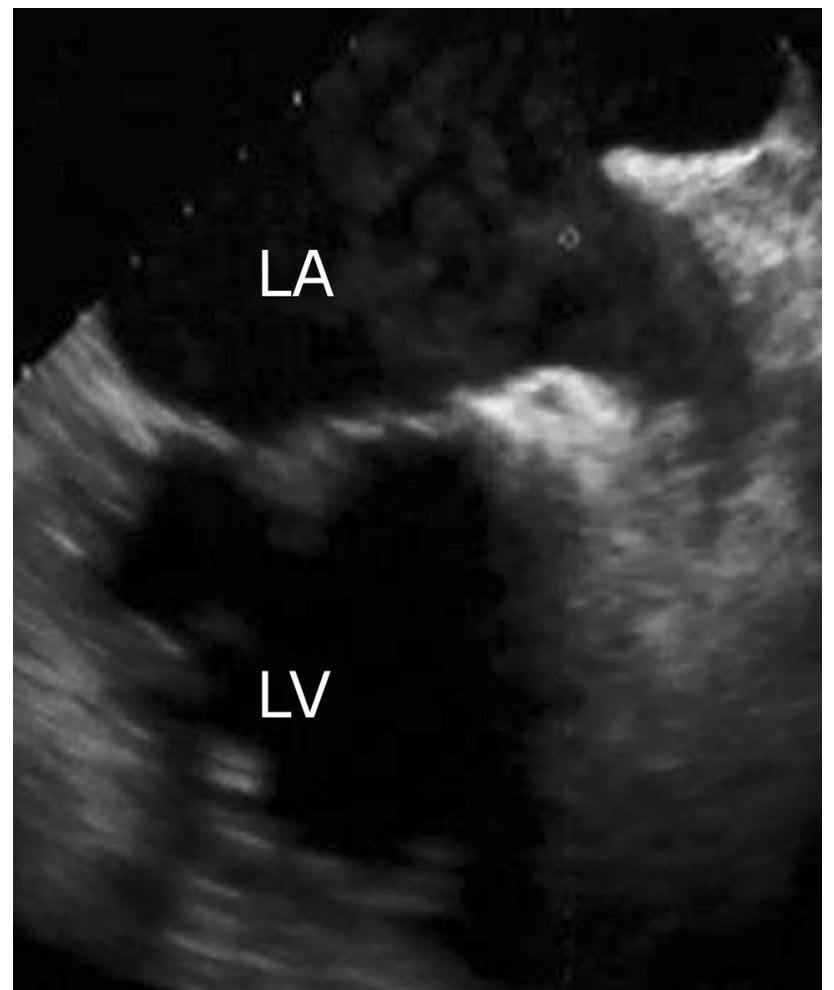

Fig. 1 Intra-operative transesophageal echocardiogram shows "Chicken Wing" left atrial appendage. $L A$ left atrium, $L V$ left ventricle

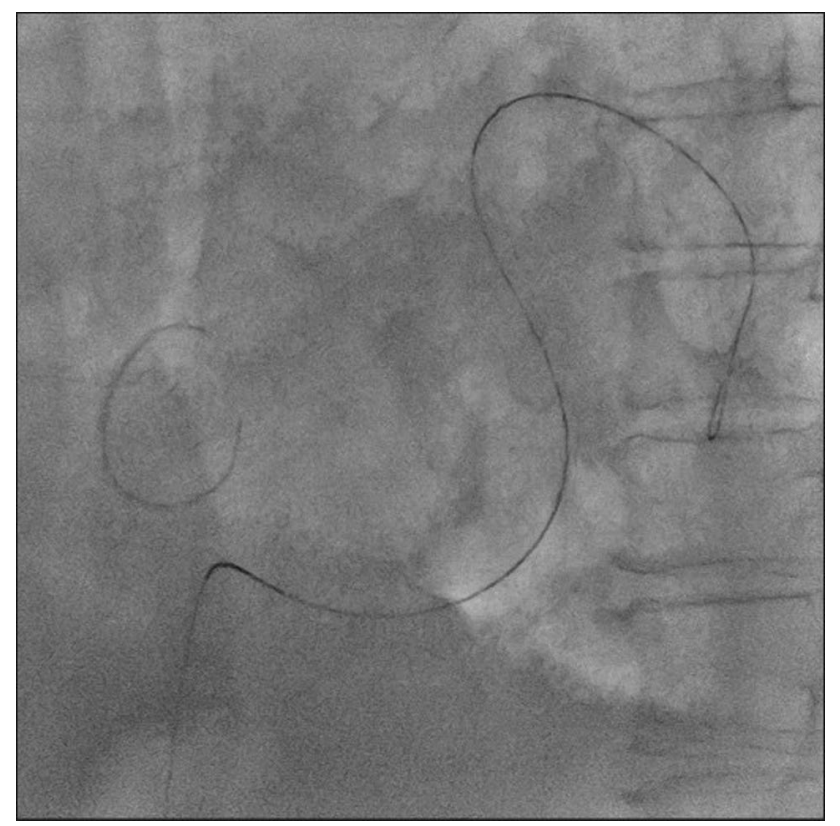

Fig. 2 A guidewire inserted through the drainage catheter shows a tortuous course inside the pericardial sac and routed around the left atrial appendage

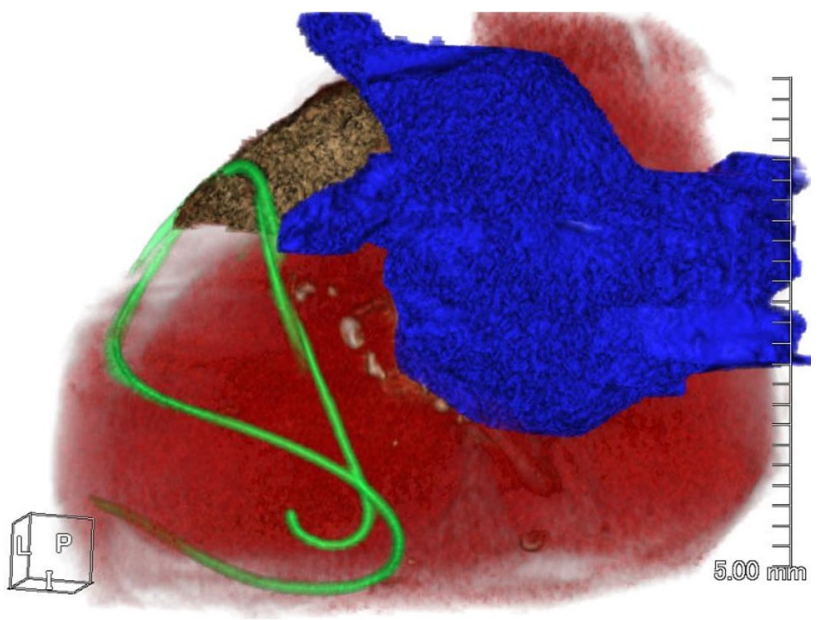

Fig. 3 Three-dimensional reconstruction of computed tomography. The catheter is seen to pass around part of the left atrial appendage. Blue indicates left atrium and pulmonary veins, brown represents left atrial appendage. Green indicates the pigtail catheter

\section{Compliance with ethical standards}

Conflict of interest The authors have no conflicts of interest to declare.

Open Access This article is distributed under the terms of the Creative Commons Attribution 4.0 International License (http://creativecommons.org/licenses/by/4.0/), which permits unrestricted use, distribution, and reproduction in any medium, provided you give appropriate credit to the original author(s) and the source, provide a link to the Creative Commons license, and indicate if changes were made.

\section{References}

1. Boersma LV, Schmidt B, Betts TR, Sievert H, Tamburino C, Teiger E, et al. Implant success and safety of left atrial appendage closure with the WATCHMAN device: peri-procedural outcomes from the EWOLUTION registry. Eur Heart J. 2016;37:2465-74.

2. Boersma LV, Ince H, Kische S, Pokushalov E, Schmitz T, Shmidt B, et al. Efficacy and safety of left atrial appendage closure with WATCHMAN in patients with or without contraindication to oral anticoagulation: 1-year follow-up outcome data of the EWOLUTION trial. Heart Rhythm. 2017;14:1302-8.

3. Di Biase L, Santangeli P, Anselmino M, Mohanty P, Salvetti I, Gili S, et al. Does the left atrial appendage morphology correlate with the risk of stroke in patients with atrial fibrillation? Results from a multicenter study. J Am Coll Cardiol. 2012;60:531-8.

Publisher's Note Springer Nature remains neutral with regard to jurisdictional claims in published maps and institutional affiliations. 